\title{
Photospheric and chromospheric activity in V405 Andromedae
}

\section{An M dwarf binary with components on the two sides of the full convection limit}

\author{
K. Vida ${ }^{1,2}$, K. Oláh ${ }^{2}$,Zs. Kővári ${ }^{2}$, H. Korhonen ${ }^{3}$, J. Bartus ${ }^{4}$, Zs. Hurta ${ }^{1,2}$, and K. Posztobányi ${ }^{5}$ \\ 1 Eötvös Loránd University, Department of Astronomy, 1518 Budapest, PO Box 32, Hungary \\ e-mail: vidakris@elte.hu \\ 2 Konkoly Observatory of the Hungarian Academy of Sciences, 1525 Budapest, PO Box 67, Hungary \\ European Southern Observatory, Karl-Schwarzschild-Straße 2, 85748 Garching bei München, Germany \\ 4 Astrophysical Institute Potsdam, An der Sternwarte 16, 14482 Potsdam, Germany \\ 5 AEKI, KFKI Atomic Energy Research Institute, Thermohydraulic Department, 1525 Budapest 114, PO Box 49, Hungary
}

Received 14 April 2009 / Accepted 30 June 2009

\section{ABSTRACT}

\begin{abstract}
We investigate the fast-rotating $\left(P_{\mathrm{orb}}=P_{\mathrm{rot}}=0.465 d\right)$, active dwarf binary V405 And $(\mathrm{M} 0 \mathrm{~V}+\mathrm{M} 5 \mathrm{~V})$ using photometric $B V(R I)_{\mathrm{C}}$ and optical spectroscopic data. The light variation is caused by the combined effect of spottedness and binarity with a small eclipse. We estimate the system parameters from the available light and radial velocity curves. Three flare events occurred during the observations: two were found in the spectroscopic data and one was observed photometrically in $B V(R I)_{\mathrm{C}}$ colours. An interesting eruptive phenomenon emerged from the photometric measurements that can be interpreted as a series of post-flare eruptions lasting for at least 3 orbits (rotations) of the system, originating from trans-equatorial magnetic loops, which connect the active regions in the two hemispheres. The two components of V405 And have masses well over and below the theoretical limit of full convection. This rare property makes the binary an ideal target for observing and testing models for stellar dynamo action.
\end{abstract}

Key words. stars: individual: V405 Andromedae - binaries: eclipsing - stars: activity - stars: chromospheres - starspots stars: fundamental parameters

\section{Introduction}

Low-mass stars are the focus of stellar structure studies. These kind of objects are the majority in the Galaxy; yet, because of their low luminosity, it is not easy to study them in detail. Low-mass stars with fast rotation show magnetic phenomena (spots, plages, flares, strong X-ray emission) due to some kind of magnetic dynamo in their interiors. Stars with a radiative core and convection zone are thought to operate $\alpha \Omega$ dynamos. The $\alpha$-effect creates a poloidal field from a toroidal one by helical turbulence in the convection zone, whereas the $\Omega$-effect produces toroidal field from the poloidal one by differential rotation. The place of the dynamo process is thought to be a thin layer between the core and convection zone, called the tachocline, where the shear is the strongest. Below about $\sim 0.32 M_{\odot}$, stars are thought to be fully convective, yet, show all the known magnetic phenomena as well. The source of the magnetic field in these low-mass stars could be the $\alpha^{2}$ dynamo generating strong, longlasting, axisymmetric magnetic fields by turbulence, or by some other mechanism involving differential rotation. However, the mass limit (or the spectral limit of M3.5) of the full convection is not well-established, since strong magnetic fields may shift this boundary towards lower masses (Mullan \& MacDonald 2001; Chabrier et al. 2007). For low-mass ( $M<0.8)$, active stars, a significant discrepancy is found between the models and observations of stellar radii; i.e., theory predicts $\sim 10 \%$ lower radii, while temperatures are underestimated by about $5 \%$ (see e.g. Ribas 2006)

An interesting approach is to study low-mass stars above and below this hypothetical mass limit together in close binary systems. To our knowledge, only two such systems are known. 2MASS J04463285+1901432, in NGC 1647 (Hebb et al. 2006) has an orbital period of $0.619 d$ and masses of 0.47 and $0.19 M_{\odot}$; however, its $V=19.34$ prevents more detailed investigation of this binary.

The binary studied in this work is an X-ray bright object, listed in the ROSAT All-Sky-Survey as RX J0222.4+4729 (Voges et al. 1996), later named V405 And and classified as RS CVn type in the General Catalogue of Variable Stars (Kazarovets et al. 2000; Samus et al. 2009). The only detailed study of V405 And is from Chevalier \& Ilovaisky (1997) (CI97 hereafter), who find that the binary has an orbital period of $P=$ $0.465 d$ showing a small (near grazing) eclipse and present photometric observations in $B$ and $V$, radial velocity data, and an $\mathrm{H} \alpha$ study. The components have spectral types M0V and M5V derived by CI97, both of them showing strong $\mathrm{H} \alpha$ emission lines, whose primary is modulated by the rotation. The original classification of the system is as BY Dra binary by the above authors. The definitions of the RS and BY types overlap in the GCVS, and both classifications seem to be valid for V405 And. In CI97, orbital elements and estimates of the masses around 0.2 and $0.5 M_{\odot}$ and radii of 0.78 and $0.25-0.30 R_{\odot}$ are given as well, which were used as the starting point for our analysis.

\section{Observations and data reduction}

Photometric observations were carried out at the $1 \mathrm{~m}$ RCC telescope of Konkoly Observatory at Piszkéstetô Mountain Station equipped with a Princeton Instruments $1300 \times 1300$ CCD and the 


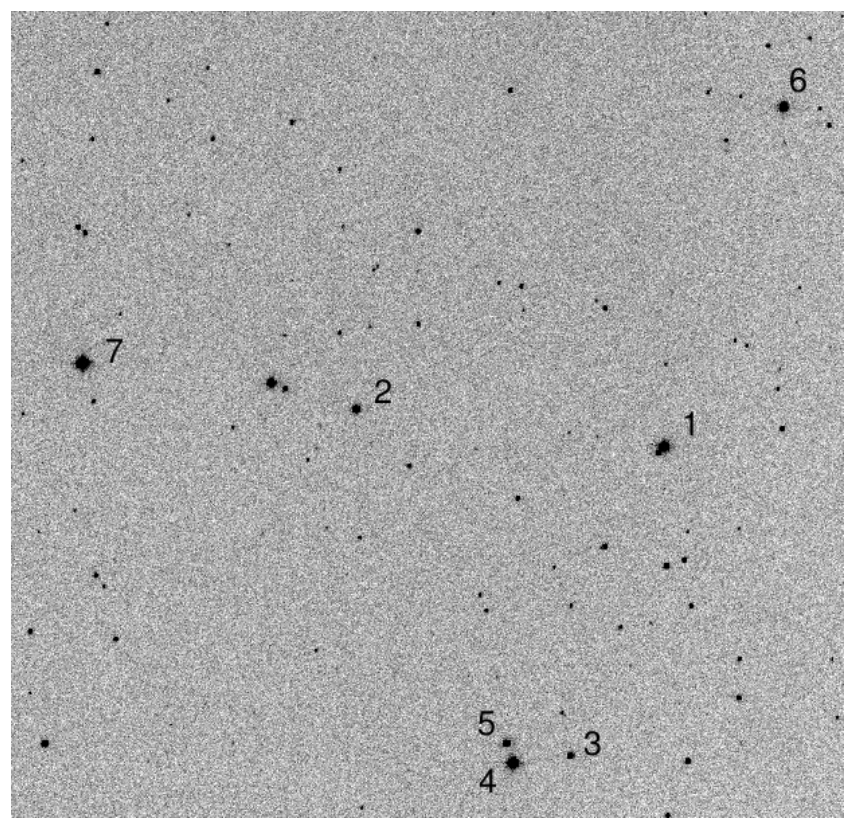

Fig. 1. Finding chart for V405 And from a $V$-band frame of the $1 \mathrm{~m} \mathrm{RCC}$ telescope. Numbering is the same as in Chevalier \& Ilovaisky (1997).

$60 \mathrm{~cm}$ telescope of Konkoly Observatory at Svábhegy, Budapest with a Wright Instruments $750 \times 1100$ CCD camera.

Observations were collected during 28 nights: 13 nights with the $60 \mathrm{~cm}$ telescope (February 8-October 1, 2007; 2454140-2454375 HJDs), and 18 nights with the RCC telescope (September 28, 2007 - November 18, 2008; 24543722454789 HJDs). On three nights both telescopes were used simultaneously. After correcting for atmospheric extinction and transforming the data to standard photometric system, we matched the two data series by fine-tuning the telescope constants within their errors.

A finding chart is plotted in Fig. 1, V405 And is marked as Star 1 (using the numbering of C197). Two of the comparison stars (Star 3, Star 6) used by CI97 turned out to be variable. Star 3 shows changes on a longer timescale of 20-30 days. Star 6 , showing a period of $\sim 0.658 d$, is possibly a W UMa type star. This effect would only cause small shifts, if any, in CI97, since they used the average value of the five comparison stars.

In this work GSC 03298-00148 (Star 7) was used as a comparison star. Magnitudes of Star 7 were calculated using stars 2, 3,4 , and 5 separately and the results compared. The differences were less than $0.03 \mathrm{mag}$. According to the results, we chose finally Star 2 for defining the magnitudes of the comparison star.

As mentioned in C197, there is a star near V405 And, at about 4.5 arc-seconds to the south-east, which is blended with the variable on most of the frames. We determined the magnitudes of the star near V405 And using frames taken on 2007 September 29, October 01, and October 02. IRAF ${ }^{1}$ PHOT routine of DAOPHOT package with a small aperture, and NSTAR PSF-fitting routine were used. There was a systematic difference (order of $0.01 \mathrm{mag}$ ) between aperture and PSF photometry (aperture photometry gave brighter magnitudes). Since the two stars are only separated by 12.6 pixels, V405 And extends

\footnotetext{
${ }^{1}$ IRAF is distributed by the National Optical Astronomy Observatory, which is operated by the Association of Universities for Research in Astronomy, Inc., under cooperative agreement with the National Science Foundation.
}
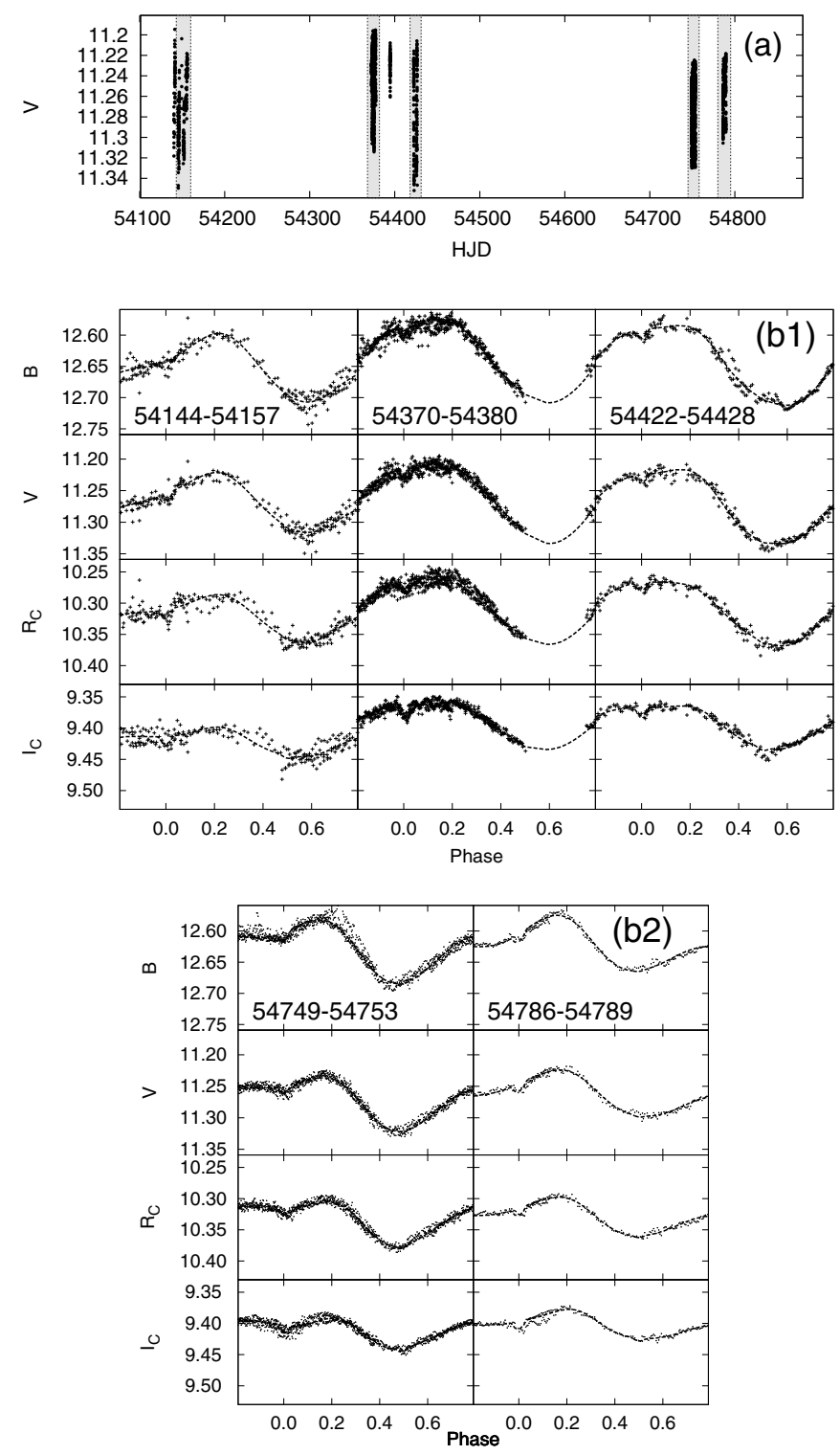

Fig. 2. a) Light curve in $V$ passband. The scale is smaller than the actual changes of the star caused by a flare at the beginning of the observation. b1,b2) Four-colour light curves of V405 And, and the fitted light curve solution containing both binarity and spottedness. The phased light curves contain observations from Julian date intervals shown on the plots.

into the aperture of the fainter star, so the result of the PSF photometry was accepted. The magnitudes of the faint star are $16.52 \pm 0.06,14.92 \pm 0.05$ (15.1 in CI97), $13.75 \pm 0.03$, and $12.13 \pm 0.03$ in $B, V, R_{\mathrm{C}}$, and $I_{\mathrm{C}}$ passbands, respectively. To test the correctness of these values, we compared PSF-magnitudes of V405 And with magnitudes from aperture photometry. We set an aperture large enough to contain both V405 And and the faint star, then subtracted the intensity of the faint star from the result. These magnitudes from aperture photometry and PSFphotometry match reassuringly within a few hundredths of magnitude.

Differential aperture photometry of V405 And was done using DAOPHOT routine. The aperture was chosen to contain the faint star on all frames, since the two stars were not resolved on most frames. The intensities of this star were subtracted from the 
Table 1. Average magnitudes of stars on V405 And field.

\begin{tabular}{lcccc}
\hline \hline Star No. & $V$ & $B-V$ & $V-R_{\mathrm{C}}$ & $V-I_{\mathrm{C}}$ \\
\hline 1 (V405 And) & 11.25 & 1.36 & 0.94 & 1.86 \\
& 11.10 & 1.44 & 0.89 & 1.74 \\
2 & 12.80 & 0.43 & 0.28 & 0.55 \\
& 12.80 & 0.43 & 0.28 & 0.55 \\
3 & 13.60 & 0.43 & 0.28 & 0.57 \\
& 13.60 & 0.41 & 0.28 & 0.54 \\
4 & 11.05 & 0.44 & 0.28 & 0.54 \\
& 11.05 & 0.43 & 0.28 & 0.54 \\
5 & 12.99 & 0.89 & & \\
& 12.96 & 0.94 & & \\
6 & 11.67 & 0.68 & 0.45 & 0.83 \\
& 11.65 & 0.72 & 0.42 & 0.79 \\
7 & 10.67 & 0.76 & 0.47 & 0.87 \\
\hline
\end{tabular}

Values in italics are from CI97, Star 7 is not in the field of view in CI97.

light curve after transforming to the standard Johnson-Cousins photometric system. The resulting light curve is plotted in Fig. 2.

Interstellar extinction was checked for V405 And using NASA/IPAC Extragalactic Database (NED), giving a value of $E(B-V)=0.182 \mathrm{mag}$ for total extinction. According to CI97, the distance of V405 And is $25-35$ pc, so the effect of interstellar extinction would only be a few thousandths of a magnitude. This value is much lower than the errors of the photometry $(\sim 0.01-$ $0.02 \mathrm{mag}$ ), so it can be neglected.

Altogether, 97 high-resolution spectra were retrieved from the ELODIE archive at the Observatoire de HauteProvence (OHP) (Moultaka et al. 2004). In 1994, 38 spectra were observed: 27 between October 18-23 and 11 between December 12-16. Another 59 unpublished spectra were obtained between October 25-30, 1998. The spectra from the archive cover the wavelength range of 4000-6800 $\AA$ with a resolution $(\lambda / \Delta \lambda)$ of 42000 .

\section{Analysis}

Results in Table 1 show that, while the average magnitudes of our measurements agree quite well with those of CI97 in the case of Stars 2 to 6, values for V405 And seem to differ significantly. V405 And was 0.15 mag fainter during our observations in $V$ band than in 1995. This and the decrease of $V-R_{\mathrm{C}}$ and $V-I_{\mathrm{C}}$ indices indicate greater spottedness. In the same time, the bluer $B-V$ show the presence of more faculae. Thus, we conclude that the activity is enhanced compared to the 1995 level.

\subsection{Spots: photometric modelling}

For finding the light curve solution, we adopted the method described in Strassmeier et al. (2008). The method uses an iterative modelling for the binarity and spottedness separately. The binary model was computed using PHOEBE (Prša \& Zwitter 2005) simultaneously for our four-colour measurements and the radial velocity curve from CI97. The binary model light curve is removed from the observed one using the following equation:

$F_{\text {spot }}=F_{\text {obs }}-F_{\text {model }}+F_{\text {unsp }}$,
Table 2. Binary parameters from the system modelling and spot model parameters.

\begin{tabular}{lc}
\hline \hline$P_{\text {orb }}=P_{\text {rot }}=0.46543 d^{*}$ & $B_{\text {unsp }}=12.42$ \\
$\mathrm{a}=2.246 R_{\odot}{ }^{* *}$ & $V_{\text {unsp }}=11.00$ \\
$q=0.42 \pm 0.01$ & $R_{\text {unsp }}=10.07$ \\
$i=66.5 \pm 1^{\circ}$ & $I_{\text {unsp }}=9.17$ \\
$M_{1}=0.49 M_{\odot}$ & $T_{\text {spot }}=3300 \mathrm{~K}$ \\
$M_{2}=0.21 M_{\odot}$ & \\
$T_{1}=4050 \pm 200 \mathrm{~K}$ & \\
$T_{2}=3000 \pm 300 \mathrm{~K}$ & \\
$R_{1}=0.78 \pm 0.02 R_{\odot}$ & \\
$R_{2}=0.24 \pm 0.04 R_{\odot}$ & \\
$v \sin i=85 \mathrm{~km} \mathrm{~s}^{-1 *}$ & \\
$e=0.0^{*}$ & \\
\hline
\end{tabular}

* Adopted from CI97; ** $a \sin i$ adopted from CI97.

where $F_{\text {spot }}$ is the flux of the primary changing due to spottedness, $F_{\text {obs }}$ the observed light curve, $F_{\text {model }}$ the binary model constructed with PHOEBE, and $F_{\text {unsp }}$ the unspotted flux of the system (for model parameters see Table 2). Note, that $F_{\text {spot }}$ is a light curve that contains only the changes caused by the spottedness and is free of the effects of binarity (eclipse and changes by the distorted shape of the stars). The true spotted light curve was modelled with SPOTMODEL (Ribárik et al. 2003), which uses the analytic approach with circular spots of Budding (1977). The circumpolar part of the spotted region accounts for the longterm, overall brightness change, whereas spotted parts outside this region cause the rotational modulation. The latitude of the spots is, at least partly, determined by the ratio of buoyancy and Coriolis-force. On fast-rotating stars the Coriolis-force is dominant, which moves the spots to high latitudes (Choudhuri \& Gilman 1987). While spot models can determine spot longitudes quite reliably, photometric observations have very low information content on spot latitudes (see e.g. Kővári \& Bartus 1997). Assuming circular spots on the surface is an approximation with few parameters. Sunspots and active nests on the Sun give examples of circular active regions. By "spots" we mean regions with dark spots and bright faculae, and the (average) temperature of the region is made up from the cool and hot parts, relative to the photosphere.

We assumed two spotted regions on V405 And with average $T_{\text {spot }}=3300 \mathrm{~K}$ on the high-latitude regions of the primary. Tests of Kôvári \& Bartus (1997) have shown that analytic models using two circular spots describe the observed spotted light curves satisfactorily within the precision of the photometric observations. The spot temperature was chosen by modelling a $V-I_{\mathrm{C}}$ light curve, and the result was accepted for all passbands. For finding the best-fitting spotted light curve, the size and position of the spots were searched. During our observations, the star was more than 0.1 magnitudes fainter due to higher spottedness than the faintest observed value in CI97, so we used the brightest part of the $V$ and $B-V$ light curves of CI97 as the (faintest possible) unspotted $B$ and $V$ magnitudes. Unspotted $R_{\mathrm{C}}$ and $I_{\mathrm{C}}$ magnitudes were calculated from the colour indices. The resulting spotted curve was then removed from the observed one, and these steps were repeated until we got a satisfactory fit for the spots and binarity (Fig. 3) separately, and for the observed light curve itself (Fig. 2b).

Using this modelling method it is possible to determine the physical parameters of the binary (see Table 2). For the initial values of surface temperatures we used the tables of Flower (1996) and VandenBerg \& Clem (2003). The shape of the light 


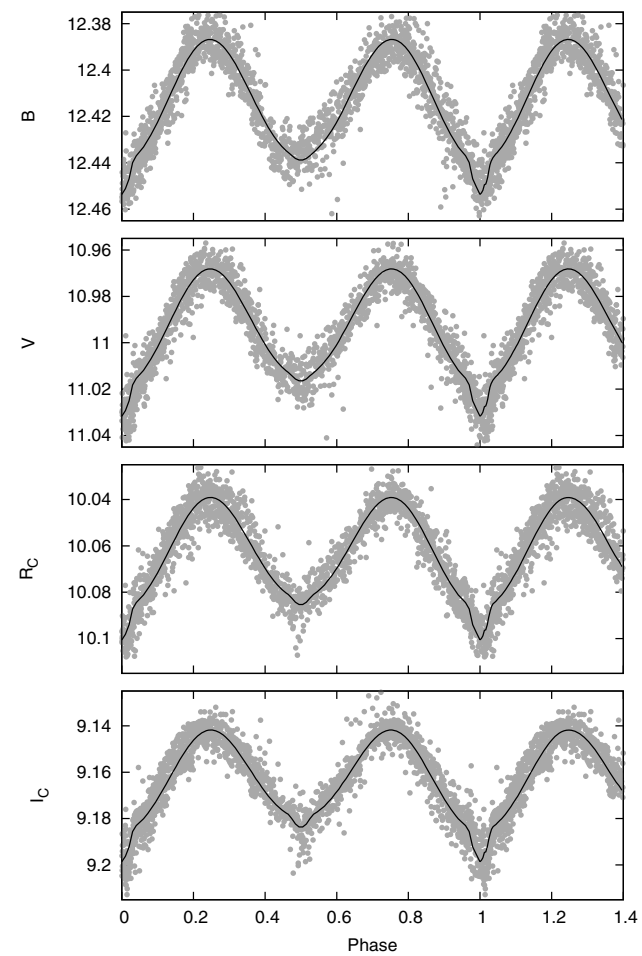

Fig. 3. Phased $B V(R I)_{\mathrm{C}}$ light curves from 2007 after subtracting the spot model from the observations. This dataset was used for determining the binary system parameters with PHOEBE.

curve is not very sensitive to the changes in the temperature, so fitting these values is quite uncertain. Our result for the mass ratio is within $1 \sigma$ of the value that was spectroscopically determined in CI97 $(q=0.38 \pm 0.04)$.

The spot configuration is relatively stable throughout the observations, so that the positions and sizes of the spots change only slightly. According to the model, one spot is situated in the northern hemisphere, and another one in the southern hemisphere about $150^{\circ}$ from each other in longitude. From the modelling results, the longitudes of spots (i.e., phases) are accurate within a few degrees, but latitude results are just approximations. Using the supposed $T_{\text {spot }}$, we find that about $25 \%$ of the stellar surface is covered with active regions. The stability of circular spot models are discussed in detail in Kôvári \& Bartus (1997). With the help of this simple spot model, we could satisfactorily separate the light variation originating from binarity and activity.

\subsection{Hot regions in the chromosphere and photosphere}

The chromospheric activity of V405 And was investigated using $\mathrm{H} \alpha$ line profiles. Equivalent widths (Fig. 4) were measured by fitting Gaussian profiles using the SPLOT task in IRAF, after the continuum was fitted by polynomials and removed. The equivalent widths vary between $1-2 \AA$ and show a small rotational modulation. This variation seems to be fairly regular during the observations, except for the time of the flares in 1994 October and 1998 (see Sect. 3.3). The observed variation indicates a permanent plage structure in the stellar atmosphere.

In 2007 September 29-30, an interesting phenomenon was observed (see $B V(R I)_{\mathrm{C}}$ light curves in Fig. 5). During two consecutive nights at the beginning of the observations, the binary showed excess brightness in all bandpasses, which gradually decreased to the unspotted flux. After about a one third rotation, the

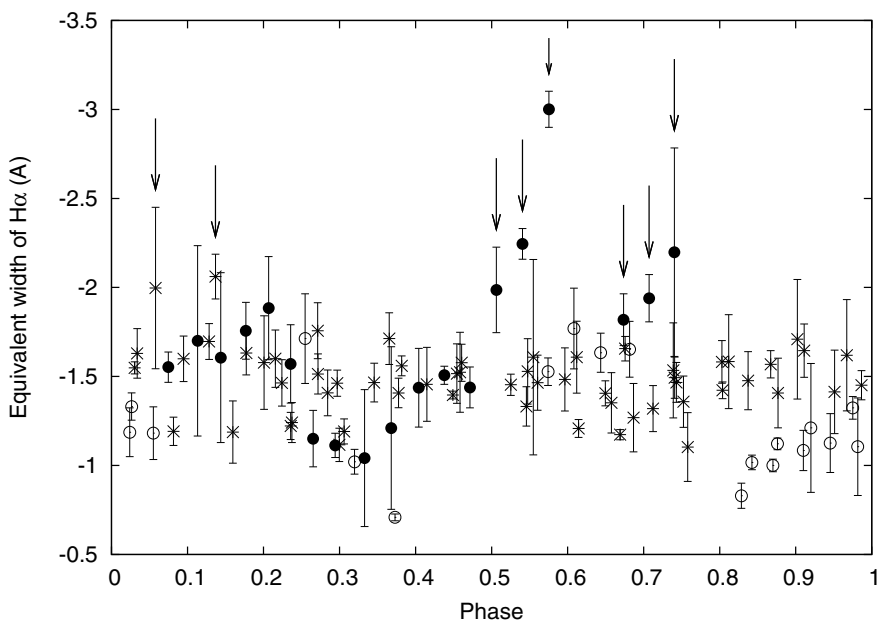

Fig. 4. Equivalent widths of the $\mathrm{H} \alpha$ line. Data obtained on 1994 October 18 are plotted with filled points, empty points show measurements from 1994 October 21-23 and 1994 December, spectra from 1998 are plotted with crosses. Arrows show times of flares in 1994 and 1998 (see Sect. 3.3).

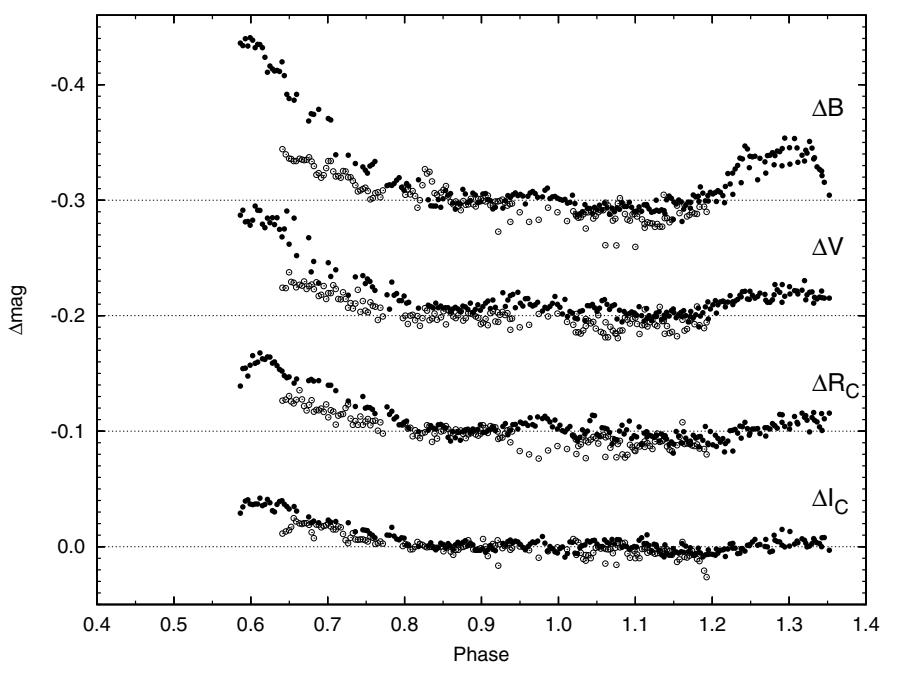

Fig. 5. Light curve observed on 2007 September 29-30 at Piszkéstető. The binary and spot model is removed from the observed light curves. Dashed lines show zero level for each passband. Data from the 29 and 30 September are plotted with empty and full circles, respectively.

brightening started again. The system configuration during the event is plotted in Fig. 6. The excess brightness was stronger during the second night (with one full rotation between the two sets of observations). The ratios of the peak intensities in $B V(R I)_{\mathrm{C}}$ filters at the beginning of the observations differ from what is produced by a usual flare event at or near its peak intensity.

Assuming that the brightening is caused by hot regions on the star, we can make a model of the surface using SPOTMODEL. Results show that two hot areas separated in longitude by $~ 90-$ $120^{\circ}$ describe the observed changes. Making use of two passbands, from $B-V$ and $V-I_{\mathrm{C}}$ indices we get hot spot temperatures of $5130 \pm 270 \mathrm{~K}$ and $5680 \pm 300 \mathrm{~K}$, respectively, which means these regions are at least $1000 \mathrm{~K}$ hotter than the surrounding area. At maximum of the brightness $B-V=1.31$ yields $4300 \mathrm{~K}$ for the temperature (Flower 1996), meaning that the whole 


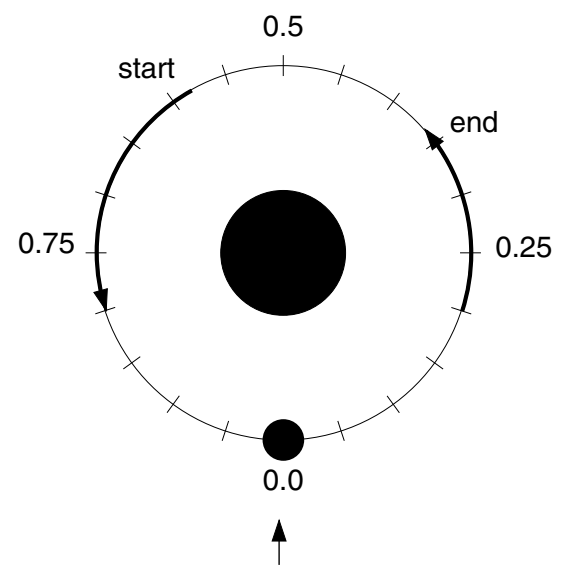

Fig. 6. Schematic plot of the eruption times and visibility on 2007 September 29-30. Arrow at the bottom shows the line of sight. The figure is plotted on the appropriate scale.

visible stellar surface seemed hotter by $\sim 150 \mathrm{~K}$, i.e.; about 10 $12 \%$ of the visible surface of the star is covered with hot regions.

An explanation of the phenomenon could be a longlasting (at least 1.5 days or 3 rotations) flare event on V405 And. During the first night's observations we saw only the decreasing part of a giant flare that might have occurred during daytime, before the observations started. At the beginning of the second night we observed a peak that could then be a post-flare eruption. Two smaller eruptions happened later that night, the first small, the second more powerful and it started to decrease before the end of the night.

A similar longlasting event has been observed on AU Mic. This event was described and modelled in Katsova et al. (1999). Comparing their results with observed solar phenomena, the authors supposed longlasting, giant post-flare loops with a size of 1-2 stellar radii and an energy balance through continuing reconnections.

Transequatorial magnetic loops are common features on the Sun. Harra et al. (2003) studied the energy mechanism of such a solar loop system and found brightenings and flare-like events during 2 days of observations. On V405 And from photometric observations, we suggest a 1-1 dominant active region in both hemispheres of the primary separated by $\sim 150^{\circ}$ in longitude. We could explain the observed phenomenon on 2007 September 2930 with a transequatiorial loop system that causes the brightenings and the flare-like events. As the star rotates, the emitting part of the loop system eventually turns in and out of view. This scenario would explain the turnbacks of the light curve seen, e.g., in $B$ band at 0.3 phase (Fig. 5), and also the intensity ratios in different filters, which are comparable to the post-flare event of Fig. 7.

\subsection{Flares}

During the observations, three noteworthy flare events occurred. Two of them were observed with the ELODIE spectrograph on 1994 October 18 and on 1998 October 29. The third one occurred on 2007 February 9 and was detected in $B V(R I)_{\mathrm{C}}$ photometric bandpasses.

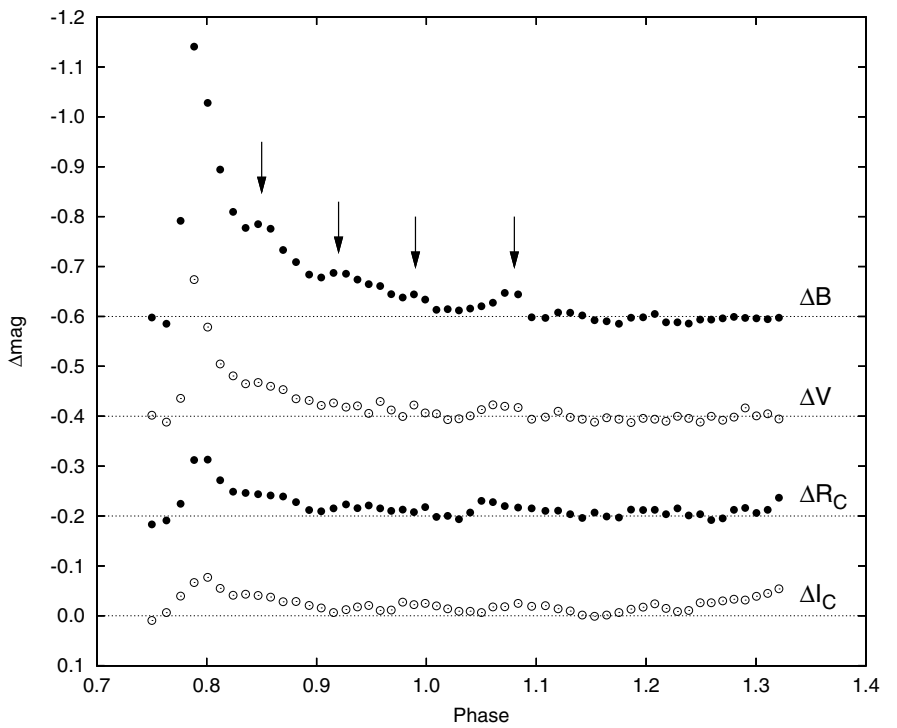

Fig. 7. Flare observed on 2007 February 9 in Budapest. The binary and spot model is removed from the observed light curves. Light curves from up to down are made using $B, V, R_{\mathrm{C}}, I_{\mathrm{C}}$ filters. Dashed lines show the zero level for each passband (with an arbitrary shift applied for better visibility). Arrows show the times of the post-flare events.

\subsubsection{Spectroscopic events}

The 1994 flare was visible in the $\mathrm{H} \alpha, \mathrm{H} \beta$, and $\mathrm{He} \mathrm{I} \mathrm{D}_{3}$ lines. During the flare, the $\mathrm{H} \alpha$ equivalent widths increased (see Fig. 4) indicating stronger activity in the chromosphere. On the dynamic spectrum (Fig. 8, left panel), as well as on the individual spectra (Fig. 9, left panel), enhancement in the blue wing of the $\mathrm{H} \alpha$ line was observed from the beginning of the observations. The flare eruption started at phase 0.51 , reached its maximum at phase 0.58 , and lasted for more than $2.5 \mathrm{~h}$, at least until the end of the observations. At the time of the flare $\mathrm{H} \beta$ is also increased, and asymmetry is seen in the red wing at all phases (Fig. 9 middle panel). As the indicator of the explosive event, He $\mathrm{I} \mathrm{D}_{3}$ appears as a prominent emission line (Fig. 9, right panel) at phases $0.54-0.58$. A very similar event is reported on LO Peg by Eibe et al. (1999).

The weaker flare from 1998 October 29 shows increased $\mathrm{H} \alpha$ emission, and the $\mathrm{H} \beta$ level is also higher, but only a marginal $\mathrm{He} \mathrm{ID}_{3}$ emission is seen (see Fig. 11). This weaker flare was not accompanied by a (large) prominence, and the $\mathrm{H} \alpha$ line is quite symmetric, except before and during the flare at phases $0.06-$ 0.14 (Fig. 8, middle panel). The $\mathrm{H} \alpha$ line outside the small flare is the same as in the quiescent $\mathrm{H} \alpha$ state (Fig. 8, right panel). As in 1994 , the $\mathrm{H} \beta$ line is increased in the red wing at all phases.

The increase in the blue wing of the $\mathrm{H} \alpha$ line is probably the signature of prominence formation, i.e. emerging material in the magnetic loop with an upflow of $\sim 40 \mathrm{~km} \mathrm{~s}^{-1}$ (see Fig. 10), which forms a prominence. The $\mathrm{H} \alpha$ line is again symmetric after the flare, since most of the material is heated up and leaves the loops during the fast phase of the flare eruption. The red asymmetry of the $\mathrm{H} \beta$ line could originate from the downflow of cooling material along the flux loops.

The $\mathrm{H} \alpha$ line of the secondary star is much better visible on the spectra made at the quiescent state of the binary (Fig. 12), which is partly due to the better signal-to-noise ratio of these spectra, but the less active primary at this time also does not wash out the weaker signal of the secondary. During the observations of the quiescent phase, in the beginning, some red asymmetry is 

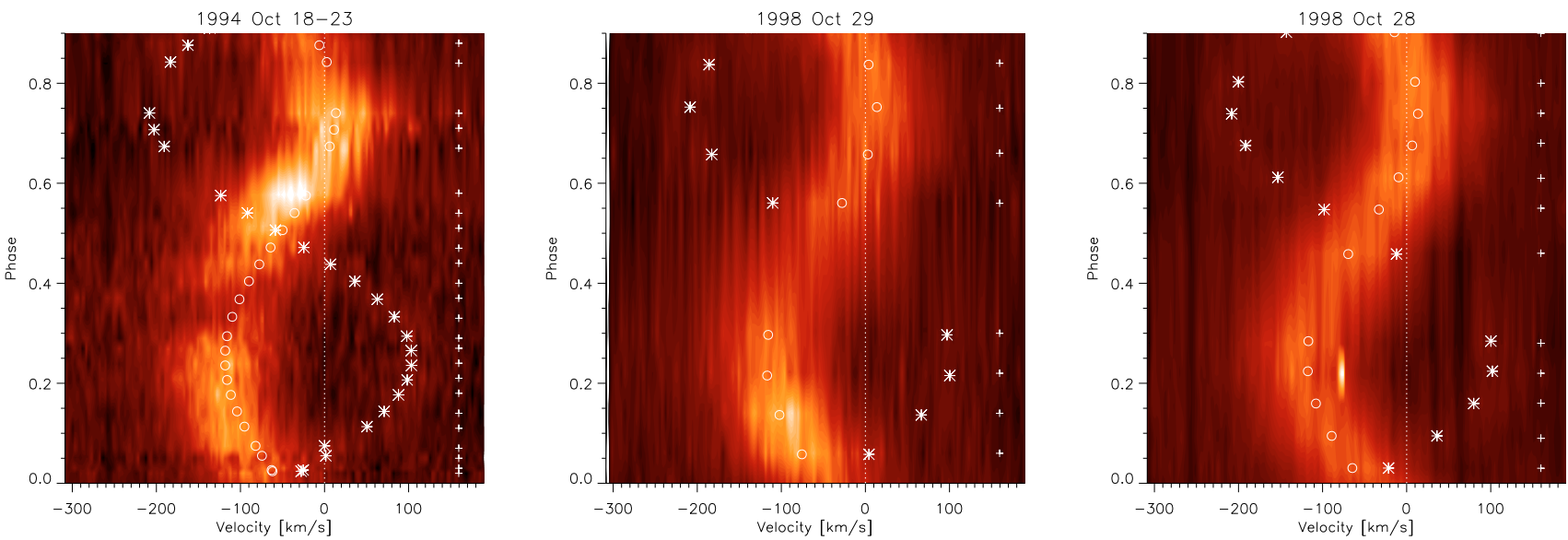

Fig. 8. Dynamic H $\alpha$ spectra from 1994 October 18 and 23, 1998 October 29 and 28 (from left to right). Crosses on the right show the observation times, circles on the plot and stars mark the radial velocity curve of the primary and secondary, respectively. The first two images on the left show two flares, the third is from a quiescent state of the binary. All plots show phases between $0.1-0.9$. The left plot shows observations from 1994 October 18 except the last two points from 1994 October 23 after phase 0.75.
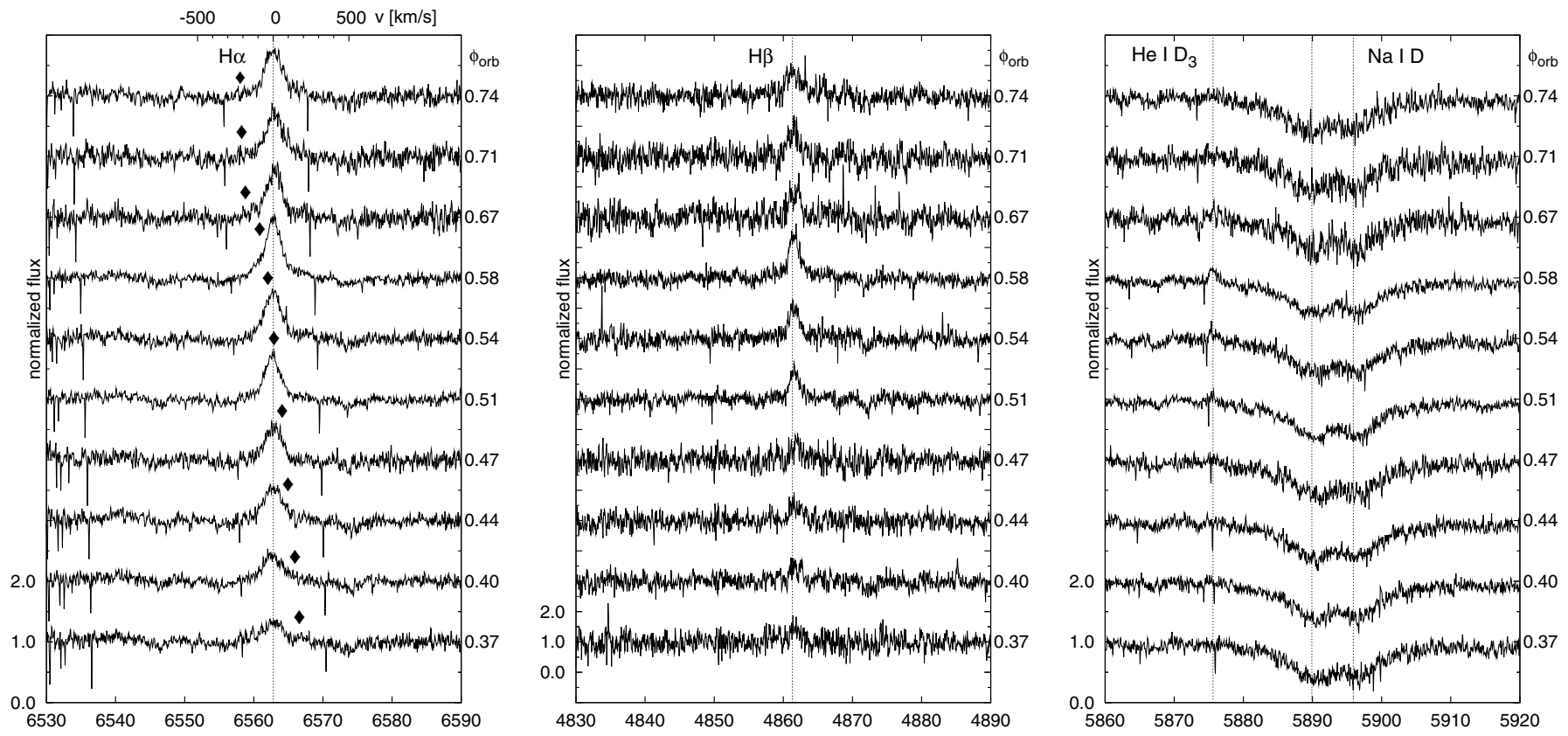

Fig. 9. $\mathrm{H} \alpha, \mathrm{H} \beta$, and $\mathrm{Na} \mathrm{D}$ lines around the time of the flare event of 1994 October 18 between phases $0.37-0.74$. The flare occurred between phases $\sim 0.5-0.7$ (see scale on the right). The scale of the $\mathrm{H} \beta$ plot is different because of the higher noise in the blue part of the spectrum. Diamonds on the $\mathrm{H} \alpha$ spectra show the place of the secondary component calculated from the radial velocity curve.

seen again in the $\mathrm{H} \beta$ line, indicating that chromospheric flows are present on the star most of the time.

\subsubsection{Photometric flare}

The light curve of the flare in February 2007 is plotted in Fig. 7. The system at the peak of the flare is 0.6 mag brighter in $B$ than the quiescent light, and the flare is also seen in $I_{C}$ filter, indicating a very strong outburst. The event could be observed for $3.72 \mathrm{~h}$, which is about one-third of the rotation. Unfortunately when using photometric data, no details can be determined for the position of the flare. It is possible that the flare lasted longer than $\sim 4 \mathrm{~h}$, but the flaring region moved out of view because of the rotation. Since both components are active, the flare could occur in either of the two components.

Assuming that the whole light curve of the flare was observed, we can estimate the flare energies. The energy emitted by the flare in $B, V, R_{\mathrm{C}}$ and $I_{\mathrm{C}}$ bands are $1.43 \mathrm{e}+35,7.58 \mathrm{e}+34$,

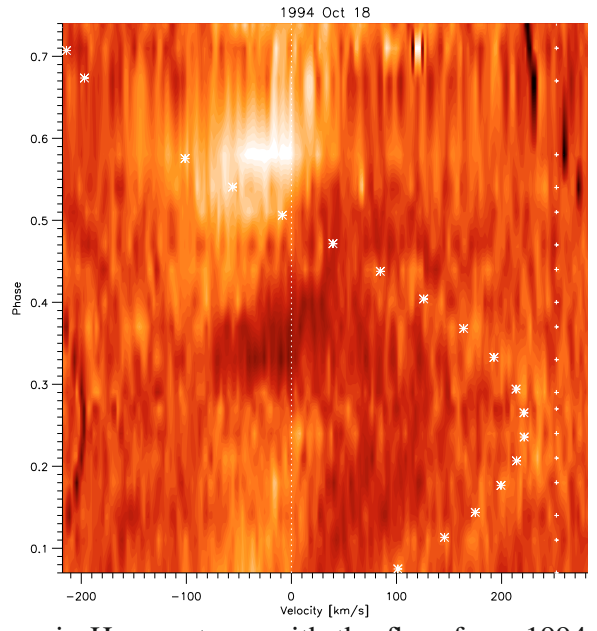

Fig. 10. Dynamic $\mathrm{H} \alpha$ spectrum with the flare from 1994 October 18 showing the excess emission compared to the average of all spectra. The spectra are shifted with their actual radial velocity. 

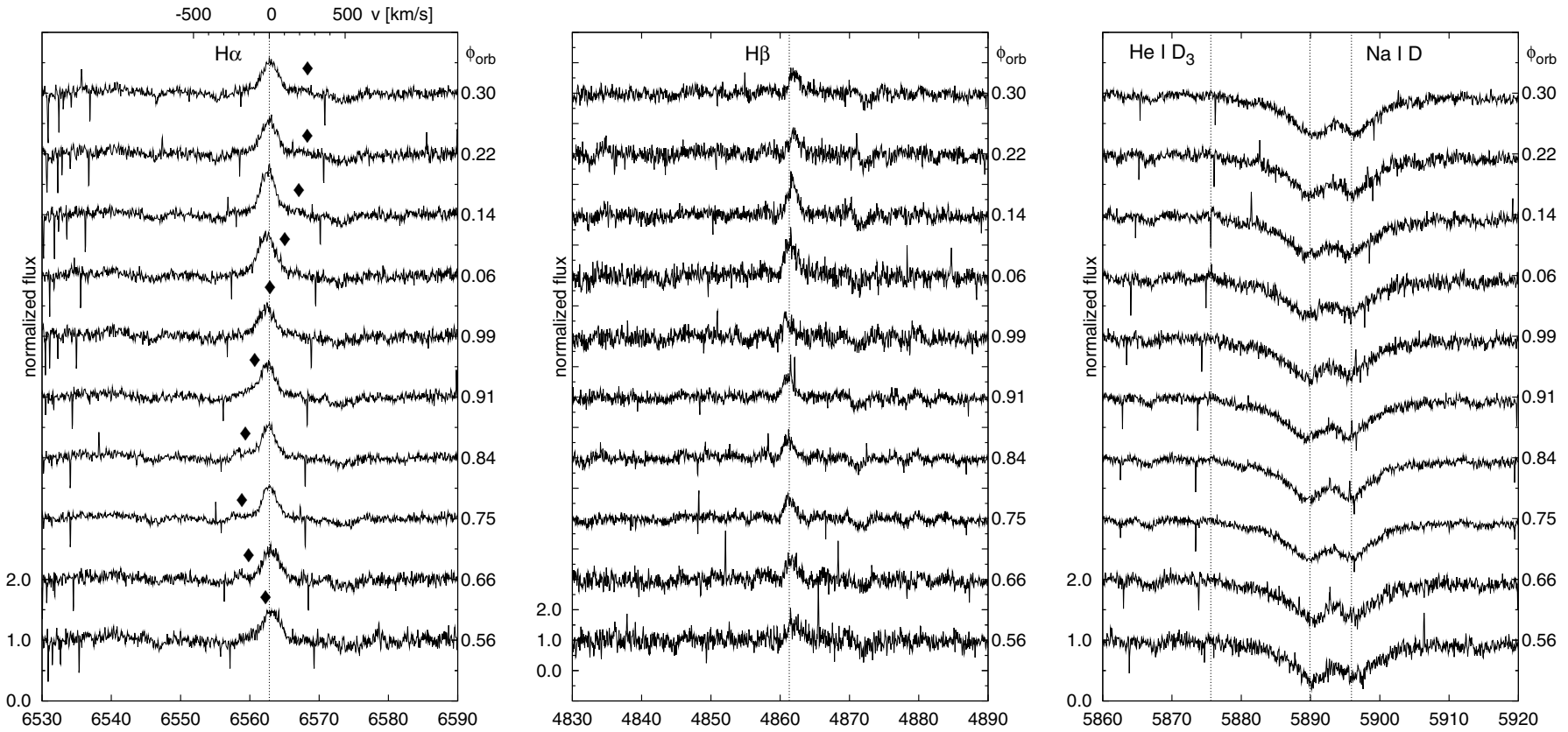

Fig. 11. $\mathrm{H} \alpha, \mathrm{H} \beta$, and $\mathrm{Na} \mathrm{D}$ lines around the time of the flare event in 1998 October 29 between phases $0.56-0.30$. The flare occurred between phases $\sim 0.05-0.15$ (see scale on the right). Scales and notation is the same as in Fig. 9.
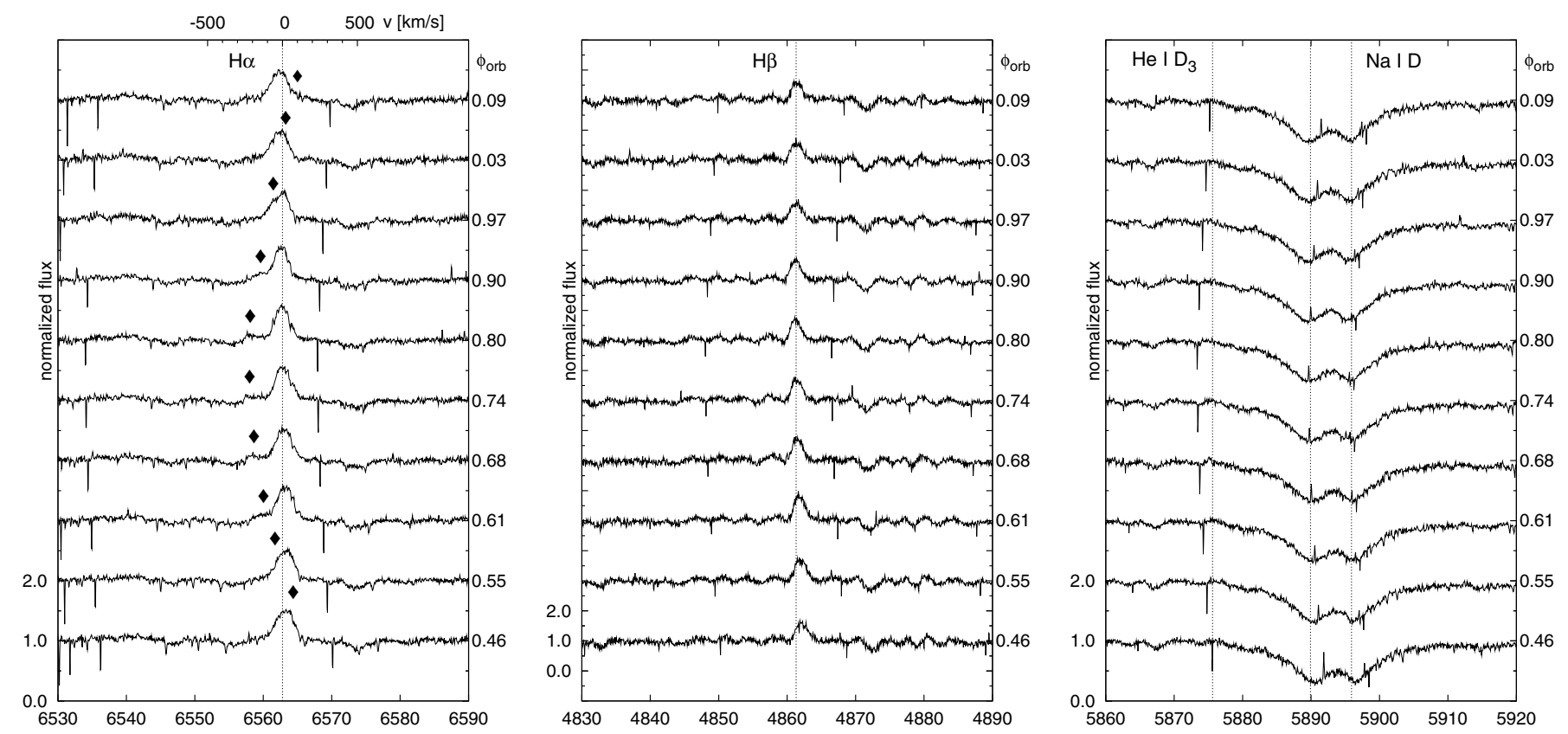

Fig. 12. $\mathrm{H} \alpha, \mathrm{H} \beta$, and $\mathrm{Na} \mathrm{D}$ lines from the quiescent state of V405 And in 1998 October 28. The contribution of the secondary can be seen around the $\mathrm{H} \alpha$ line. Scales and notation is the same as in Fig. 9.

$1.34 \mathrm{e}+35$, and $1.31 \mathrm{e}+35 \mathrm{erg}$, assuming that the flare occurred on the primary.

If we suppose that the flare occurred on the much fainter secondary, we need to know its magnitudes for calculating flare energy. According to CI97, the secondary component is at least 3 mag fainter than the primary. If we assume the solar metallicity of the system and a mass of $0.21 M_{\odot}$ from our results for the secondary, using the tables of VandenBerg \& Clem (2003), we get $B=16.1, V=14.4, R_{\mathrm{C}}=13.1, I_{\mathrm{C}}=11.5$ magnitudes as an upper limit of the secondary component's brightness. Using these values and taking the uncertainties in stellar temperatures and radii into account (see Table 2), the resulting flare energies agree satisfactorily with the values above, what we determined supposing the flare occurred on the primary. For the details of flare energy calculating, see e.g. Kővári et al. (2007).

After the peak of the flare, four smaller post-flare eruptions were observed. These oscillations could be the result of reconnection between the moving footpoints of the expanding magnetic loop causing the flare and the intermittent magnetic field (see Kővári et al. 2007; Attrill et al. 2007) or individual 


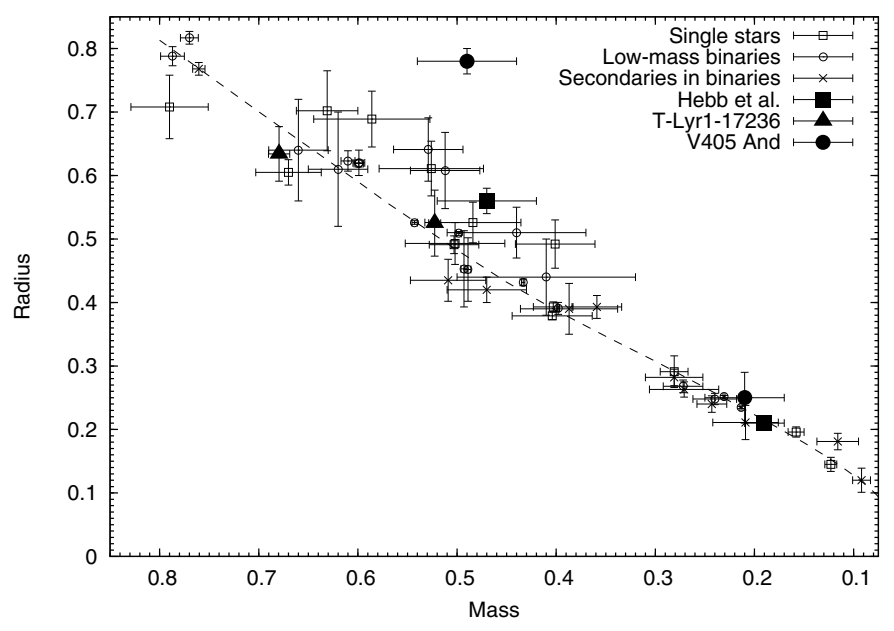

Fig. 13. Mass-radius curve for 5 Gyr from Baraffe et al. (1998) (dashed line) and observed values from López-Morales (2007), Blake et al. (2008), López-Morales et al. (2006), and Becker et al. (2008). Three systems, a binary from NGC 1647 Hebb et al. (2006), T-Lyr1-17236 Devor et al. (2008), and V405 And (present paper) are plotted with special characters

additional flares. However, the quasiperiodicity of the events makes the first scenario more likely (cf. Mitra-Kraev et al. 2005).

\section{Discussion}

Our measurements show that V405 And is a very active RS CVn binary. Strong flare events were observed both in photometry and spectroscopy (see Sect. 3.3). Photometric data show that the stellar surface is covered by large spotted regions, which are quite stable on the timescale of the observations ( $\sim 650$ day), i.e., the maximum spot visibility (spotted light curve minima) is near the same phase. The eruption on 2007 September 30 happened at this position, and probably the one on 2007 September 29 near to that. The large flare on 1994 October 18 also appears at the same phase. This evidence points towards the presence of an active nest that has a very long lifetime, more than 10 years. The phase of the eruptive events is near to the secondary minimum, so the feature is probably locked to the binary orbit. Such long-lived active regions have been observed e.g. on WY Cnc by Kozhevnikova et al. (2007). According to CI97, $\log \left(L_{\mathrm{X}} / L_{\mathrm{bol}}\right)$ for V405 And is $-3.1 \pm 0.14$, which fits the rotation-activity relations of James et al. (2000) and Pizzolato et al. (2003).

We compared the masses and radii of V405 And (Table 2) with the existing measured values of dwarf stars with masses below $0.8 M_{\odot}$ in Fig. 13. The theoretical curve for 5 Gyr from Baraffe et al. (1998) is also plotted using $T_{\text {eff, } \odot}=5780$ and $M_{\mathrm{bol}, \odot}=4.72$. At this part of the theoretical mass-radius diagram, there is not much difference between isochrones belonging to different ages; low-mass stars evolve slowly. It can be seen that stars less massive than $\sim 0.32 M_{\odot}$, which has been suggested as the limit of full convection, fit the mass-radius relation well. Among these low-mass stars are the secondary components of the binary from NGC 1647 and of V405 And. However, the correlation gets looser with increasing mass. In Fig. 13 there are three systems in which the masses of the components are very different: T-Lyr1-17236, the binary from NGC 1647 (Hebb et al. 2006), and V405 And. T-Lyr1-17236 rotates rather slowly with a period of $P_{\text {rot }} \simeq 8.4 d$, whereas the other two systems are very fast rotators (V405 And: $P_{\text {rot }} \simeq 0.465 d$, star from NGC 1647: $P_{\text {rot }} \simeq 0.619 d$ ). The radii of the primary components of the two fast-rotating systems are well above the theoretically predicted radius values, especially V405 And is much larger than expected. On the other hand, both components of the slow rotator T-Lyr1-17236 fit the mass-radius relation.

Mullan \& MacDonald (2001) find that the radii of low-mass M dwarfs, when using stellar structure codes, are larger than expected. This could stem from their strong magnetic fields, which may push the mass limit of full convection towards lower masses. Ribas (2006) compared theory with observation on masses and radii of low-mass stars (both binaries and single stars). From the high-precision results of double-lined eclipsing binaries, he finds that theory predicts smaller radii by about $10 \%$ (or higher temperatures by about $5 \%$ ) for stars in the mass range between 0.4-0.8 $M_{\odot}$. Finally, Chabrier et al. (2007) carried out evolutionary calculations on low-mass stars taking magnetic activity into account, and demonstrate that magnetic fields alter the evolution and that the spot coverage yields larger radii and lower effective temperature. However, the radius of the primary component of V405 And is even larger than the results of Chabrier et al. (2007) suggest. Future modellings with magnetic fields are needed to map the area of $M$ dwarfs in the mass-radius plane. An interesting question is what the effect would be of the magnetic interaction between a very active, fully convective star with its close, also active component in a binary system, so that the radius of the more massive component becomes even larger than recent theory predicts, while at the same time the radius of the fully convective star agrees with the prediction.

At present only two systems are known whose primaries are about 2.5 times more massive than the secondaries and one component is above while the other is below the limit of the full convection. The primary of V405 And deviates most from the expected radius of its mass from the known sample. On the other hand, the secondary of V405 And would be fully convective even in the presence of its strong magnetic field according to the calculations of Mullan \& MacDonald (2001, Fig. 1). It would be a challenge to model the evolution of those binaries whose components are on both sides of the mass limit of full convection. Of the two known examples, V405 And is the one with its $V \approx 11 \mathrm{mag}$. brightness, showing all the signatures of strong magnetic activity like spots and flares, which is suitable for high-precision observations. We recommend this binary to the attention of future investigators.

Acknowledgements. Our sincere thanks are due to A. Prša for his suggestions in binary modelling and L. van Driel-Gesztelyi for helpful discussions. An anonymous referee has helped to improve the paper considerably. The financial support of OTKA grant T-068626 is acknowledged. ZsK is a grantee of the Bolyai János Scholarship of the Hungarian Academy of Sciences. This research has made use of the NASA/IPAC Extragalactic Database (NED), which is operated by the Jet Propulsion Laboratory, California Institute of Technology, under contract with the National Aeronautics and Space Administration.

\section{References}

Attrill, G. D. R., Harra, L. K., van Driel-Gesztelyi, L., et al. 2007, ApJ, 656, L101

Baraffe, I., Chabrier, G., Allard, F., et al. 1998, A\&A, 337, 403

Becker, A. C., Agol, E., Silvestri, N. M., et al. 2008, MNRAS, 386, 416

Blake, C. H., Torres, G., Bloom, J. S., et al. 2008, ApJ, 684, 635

Budding, E. 1977, Ap\&SS, 48, 207

Chabrier, G., Gallardo, J., \& Baraffe, I. 2007, A\&A, 472, L17

Chevalier, C., \& Ilovaisky, S. A. 1997, A\&A, 326, 228

Choudhuri, A. R., \& Gilman, P. A. 1987, ApJ, 316, 788 
Devor, J., Charbonneau, D., Torres, G., et al. 2008, ApJ, 687, 1253 Eibe, M. T., Byrne, P. B., Jeffries, R. D., et al. 1999, A\&A, 341, 527 Flower, P. J. 1996, ApJ, 469, 355

Harra, L. K., Matthews, S. A., \& van Driel-Gesztelyi, L. 2003, ApJ, 598, L59

Hebb, L., Wyse, R. F. G., Gilmore, G., et al. 2006, AJ, 131, 555

James, D. J., Jardine, M. M., Jeffries, R. D., et al. 2000, MNRAS, 318, 1217

Katsova, M. M., Drake, J. J., \& Livshits, M. A. 1999, ApJ, 510, 986

Kazarovets, E. V., Samus, N. N., \& Durlevich, O. V. 2000, Information Bulletin on Variable Stars, 4870,1

Kővári, Z., \& Bartus, J. 1997, A\&A, 323, 801

Kővári, Z., Vilardell, F., Ribas, I., et al. 2007, Astron. Nachr., 328, 904

Kozhevnikova, A. V., Alekseev, I. Y., Heckert, P. A., et al. 2007, Astron. Rep., 51,932

López-Morales, M. 2007, ApJ, 660, 732
López-Morales, M., Orosz, J. A., Shaw, J. S., et al. 2006, ArXiv Astrophysics e-prints

Mitra-Kraev, U., Harra, L. K., Williams, D. R., et al. 2005, A\&A, 436, 1041

Moultaka, J., Ilovaisky, S. A., Prugniel, P., et al. 2004, PASP, 116, 693

Mullan, D. J., \& MacDonald, J. 2001, ApJ, 559, 353

Pizzolato, N., Maggio, A., Micela, G., Sciortino, S., \& Ventura, P. 2003, A\&A, 397, 147

Prša, A., \& Zwitter, T. 2005, ApJ, 628, 426

Ribárik, G., Oláh, K., \& Strassmeier, K. G. 2003, Astron. Nachr., 324, 202

Ribas, I. 2006, Ap\&SS, 304, 89

Samus, N. N., Durlevich, O. V., et al. 2009, VizieR Online Data Catalog, 1, 2025

Strassmeier, K. G., Bartus, J., Fekel, F. C., et al. 2008, A\&A, 485, 233

VandenBerg, D. A., \& Clem, J. L. 2003, AJ, 126, 778

Voges, W., Gruber, R., Haberl, F., et al. 1996, VizieR Online Data Catalog, 9011, 0 